\title{
Bricklaying and the Hermite Normal Form
}

\author{
William J. Gilbert \\ Pure Mathematics Department \\ University of Waterloo \\ Waterloo, Ontario \\ Canada N2L 3G1 \\ AMS Classifications: Primary: 15A36
}

Appeared in the American Mathematical Monthly

Vol. 100, No. 3, March 1993, pp. 242-245 
We describe a geometric interpretation of the Hermite normal form of an integer matrix in terms of tilings by bricks.

A non-singular integer $n \times n$ matrix $A$ generates an integer lattice, $L$, in $n$-space. The lattice points are the integer linear combinations of the columns of $A$. Figure 1 shows such a lattice in the plane.

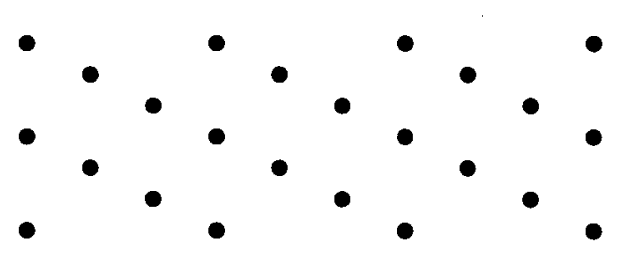

FIG. 1. A lattice generated by the columns of $\left[\begin{array}{cc}2 & 2 \\ -1 & 2\end{array}\right]$.

How can we tile $n$-space by rectangular bricks parallel to the coordinate axes so that the translation group of the tiling is the same as that of the lattice $L$ ? Figures 2 and 3 show two such tilings with the same translation group as the lattice in Figure 1. The lower left corner of each brick lies on a lattice point.

\section{Some Basic Definitions}

An integer lattice in $n$-dimensional space is the set of all integer linear combinations of $n$ linearly independent vectors with integer entries.

The translation group of the lattice consists of all the translations of $n$-dimensional space that take lattice points to lattice points. This group is isomorphic to the set of lattice points under vector addition.

A fundamental brick will be a rectangular brick with one vertex at the origin and sides along each positive coordinate axis. A brickwork will consist of a tiling of $n$-space by $n$-dimensional bricks that are translations of a fundamental brick. The set of these translations form the translation group of the brickwork.

Each lattice has many different bases. We can change the basis of the lattice $L$ by means of unimodular column operations on the matrix $A$. The elementary unimodular column operations are essentially "integer" column operations and consist of 
(i) adding an integer multiple of one column to another;

(ii) exchanging two columns;

(iii) multiplying one column by -1 .

Performing unimodular column operations on a matrix is equivalent to postmultiplying by a unimodular matrix $U$; i.e. an integer matrix $U$ whose determinant is \pm 1 . The inverse of $U$ is also an integer matrix.

Every integer matrix can be reduced, by unimodular column operations, to a unique standard form called the Hermite normal form [?, Ch. I.7], [?, Part II]. A non-singular integer matrix is said to be in Hermite normal form if it is a lower triangular, non-negative matrix in which each row has a unique maximum entry that is located on the main diagonal. (Beware that some authors call the reduced row echelon form over a field the Hermite form. Even those authors and computer packages that define the Hermite normal form over the integers, or a Euclidean ring, do so in different ways. These are all equivalent, but some use row operations instead of column operations and some use upper triangular matrices instead of lower triangular ones.)

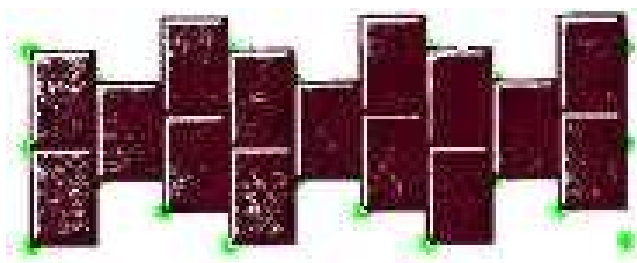

FiG. 2. Bricks of size $2 \times 3$ corresponding to the matrix $\left[\begin{array}{ll}2 & 0 \\ 2 & 3\end{array}\right]$.

For example, the lattice in Figure 1 is generated by the matrix $A=$ $\left[\begin{array}{cc}2 & 2 \\ -1 & 2\end{array}\right]$ and its Hermite normal form is $H=\left[\begin{array}{ll}2 & 0 \\ 2 & 3\end{array}\right]$. This matrix $H$ can be considered as corresponding to the brickwork in Figure 2. The diagonal elements determine the size of the bricks. The last column determines the edge along the last coordinate, while the first column determines the offset of an adjacent brick. In general, the columns of $H$ determine the lattice points on the boundary of the fundamental brick.

The Hermite normal form is obtained by unimodular reduction and so the magnitude of the determinant is unchanged. This magnitude is the 
area of a generating parallelogram of the lattice (or volume of a generating parallelepiped) and also the area (or volume) of a brick.

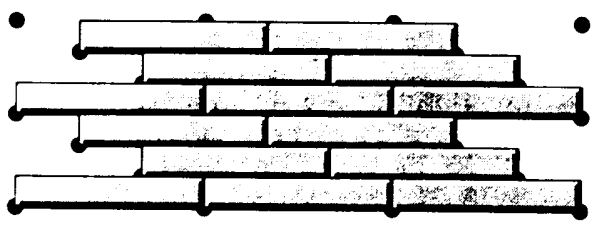

FIG. 3. Bricks of size $6 \times 1$ corresponding to the matrix $\left[\begin{array}{ll}6 & 4 \\ 0 & 1\end{array}\right]$.

What is connection between the brickwork in FiguRE 3 and the Hermite normal form? The algorithm for reducing a matrix to Hermite normal form initially consists of column reducing the matrix so that the first row is in the required form with all the off-diagonal entries zero. This first leading entry is then the greatest common divisor of all the entries in the first row. The algorithm continues inductively by leaving the first $r$ columns alone and then column reducing the others so that the last $(n-r-1)$ entries in the $(r+1)$ st row are zero. Then multiples of the $(r+1)$ st column are added to the first $r$ columns so that the $(r+1)$ st row is in the required form.

If we start with the matrix $A$ and modify this algorithm so that the second row has all off-diagonal entries zero, then we obtain the matrix $\left[\begin{array}{ll}6 & 4 \\ 0 & 1\end{array}\right]$, that corresponds to the brickwork in FiguRE 3.

For an $n \times n$ matrix $A$, there are $n$ ! ways of modifying the Hermite normal form algorithm, corresponding to the $n$ ! different orderings of the rows. Let $\pi$ be a permutation of the $n$ rows of $A$. If we inductively leave the columns $\pi^{-1}(1), \ldots, \pi^{-1}(r)$ alone and column reduce the matrix so that the other off-diagonal entries of the $\pi^{-1}(r+1)$ st row are zero, we obtain a modified Hermite normal form, $H_{\pi}$. This corresponds to a brickwork with the same translation group as that derived from $A . H_{\pi}$ is a non-negative matrix whose $(i, j)$ th entry is zero whenever $\pi(i)<\pi(j)$ and is such that each row has a unique maximum entry located on the main diagonal. If $P$ is the permutation matrix corresponding to $\pi$, then this modified Hermite normal form is

$$
H_{\pi}=P^{-1} \operatorname{hnf}(P A) P
$$

where $\operatorname{hnf}(P A)=P A U$, the Hermite normal form of $P A$, and $U$ is the 
unimodular matrix corresponding to the column operations. Notice that $H_{\pi}=A U P$ where $U P$ is a unimodular matrix. Since $P H_{\pi} P^{-1}=\operatorname{hnf}(P A)$ is lower triangular, it follows that the $(i, j)$ th entry of $H_{\pi}$ is zero whenever $\pi(i)<\pi(j)$.

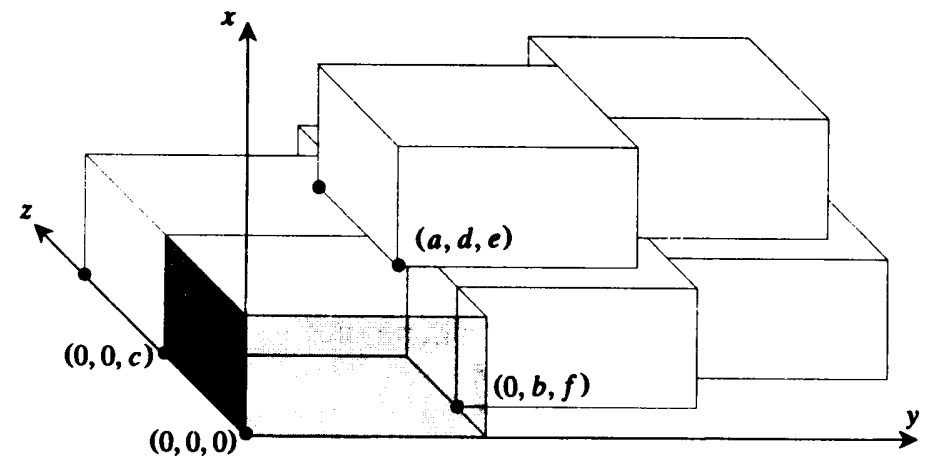

FIG. 4. Bricks of size $a \times b \times c$ corresponding to the matrix $\left[\begin{array}{lll}a & 0 & 0 \\ d & b & 0 \\ e & f & c\end{array}\right]$.

FIgURE 4 shows a typical tiling of 3 -space by bricks corresponding to the Hermite normal form of a $3 \times 3$ matrix. There are six ways of modifying this Hermite normal form. The following is an example in which the six ways give rise to brickworks that all use different sized bricks.

Consider the matrix $A=\left[\begin{array}{ccc}5 & 2 & 1 \\ -4 & 2 & 4 \\ 0 & -3 & 6\end{array}\right]$. If we perform the modified algorithm described above using the six permutations of the rows, we obtain the six matrices

$$
\begin{aligned}
& H_{(1)}=\left[\begin{array}{ccc}
1 & 0 & 0 \\
4 & 6 & 0 \\
6 & 15 & 30
\end{array}\right], \quad H_{(123)}=\left[\begin{array}{ccc}
5 & 0 & 3 \\
8 & 12 & 6 \\
0 & 0 & 3
\end{array}\right], \quad H_{(132)}=\left[\begin{array}{ccc}
15 & 8 & 6 \\
0 & 2 & 0 \\
0 & 3 & 6
\end{array}\right], \\
& H_{(12)}=\left[\begin{array}{ccc}
3 & 2 & 0 \\
0 & 2 & 0 \\
18 & 27 & 30
\end{array}\right], \quad H_{(13)}=\left[\begin{array}{ccc}
15 & 10 & 8 \\
0 & 4 & 2 \\
0 & 0 & 3
\end{array}\right], \quad H_{(23)}=\left[\begin{array}{ccc}
1 & 0 & 0 \\
4 & 12 & 6 \\
6 & 0 & 15
\end{array}\right] \text {. }
\end{aligned}
$$

These matrices all induce the same lattice in 3-space and they correspond to six brickworks that all use different sized bricks, namely $1 \times 6 \times 30,5 \times 12 \times 3$, $15 \times 2 \times 6,3 \times 2 \times 30,15 \times 4 \times 3$, and $1 \times 12 \times 15$. 
All the brickworks having the same translation group as that derived from an integer matrix $A$ arise from the modified Hermite normal forms applied to $A$. This essentially follows from the Minkowski-Hajós problem on tilings by $n$-cubes and induction on the dimension $n$. In 1907, H. Minkowski considered a problem in number theory that was equivalent to the following geometric conjecture. These problems are described in [?].

Minkowski's Conjecture. If a lattice of unit $n$-cubes tiles $n$-space, then some pair of cubes share a complete $(n-1)$-dimensional face.

In 1942, G. Hajós proved the conjecture for all $n$ by changing it into an equivalent conjecture concerning finite abelian groups.

By shrinking each axis separately, any brickwork tiling $n$-space using rectangular bricks is affinely equivalent to a tiling by unit $n$-dimensional cubes. Hence some pair of bricks must share a complete $(n-1)$-dimensional face. This $(n-1)$-dimensional face is perpendicular to one of the axes, say the $j$ th axis. If $e_{j}$ is the unit vector along this axis, then one of the edges of the bricks is along the vector $a_{j} e_{j}$, for some positive integer $a_{j}$. The lattice point $a_{j} e_{j}$ is a vertex of the fundamental brick and every brick lies a complete row of bricks parallel to the $j$ th axis, that is obtained by translating that brick by integer multiples of $a_{j} e_{j}$.

Now project the tiling onto the $(n-1)$-dimensional subspace orthogonal to the $j$ th axis. A similar argument using the Minkowski-Hajós problem in $(n-1)$-space will show that another lattice point on the fundamental brick is of the form $b_{k} e_{k}+b_{j} e_{j}$, where $k \neq j, b_{k}$ and $b_{j}$ are integers, $b_{k}>0$, and $0 \leq b_{j}<a_{j}$.

Use induction on the dimension to complete the basis for the lattice, consisting of lattice points on the boundary of the fundamental brick. This basis will form the columns of one of the modified Hermite normal forms obtained from $A$.

\section{References}

[1] G. L. Nemhauser and L. A. Wolsey, Integer and Combinatorial Optimization, Wiley, New York, 1988.

[2] A. Schrijver, Theory of Linear and Integer Programming, Wiley, Chichester, 1986.

[3] S. K. Stein, Algebraic Tiling, Amer. Math. Monthly, 81 (1974) 445-462. 